\title{
LA METODOLOGÍA GIMNÁSTICA DEL PROFESOR GARCÍA FRAGUAS
}

\section{Gymnastic methodology of Professor García Fraguas}

\author{
M. José Daniel Huerta \\ Universidad de Salamanca \\ Correo-e: marijo@usal.es \\ Fecha de envío: 25 de septiembre de 2014 \\ Envío a informantes: 4 de noviembre de 2014 \\ Aceptación definitiva: 13 de febrero de 2015
}

Resumen: En el presente artículo se recoge la propuesta didáctica que desarrolló el profesor García Fraguas en materia de gimnástica a finales del siglo xix. Para ello mostraremos el método utilizado para guardar orden y disciplina, la dinámica de sus clases, el sistema de evaluación establecido, la indumentaria recomendada, así como las reglas higiénicas propuestas entre otras.

El afán por evolucionar le llevó a visitar varias escuelas e institutos y a asistir a diversos congresos relacionados con la educación física. Esta forma de actuar, junto con su experiencia docente, fue lo que le permitió ir perfilando su metodología didáctica.

En lo que concierne a los procesos de enseñanza-aprendizaje sobre la disciplina gimnástica, hay que anotar que criticó las formas de enseñanza que se usaban en la mayor parte de las escuelas, pues aseguraba que las clases eran aburridas, por lo que no resultaban ni provechosas ni placenteras para los alumnos.

Palabras clave: Gimnástica; método didáctico; juego; segunda enseñanza; higiene y sistema de evaluación.

Aвstract: This article shows the proposal didactic developed by teacher García Fraguas on gymnastics at the end of the nineteenth century. Based on this method we are going to explain the system used to keep order and discipline, the development of the lessons, the assessment system, the suitable clothing and hygienic rules, among others.

The aim to improve the current conditions took him to visit schools and high schools and attend to different congresses related to physical education. The result of their experience was a news and original methodology.

As for as the teaching learning of the gymnastic discipline, it should be remarked that criticized the methods of teaching used in most schools because he was sure that the lessons were boring, so they were not or profitable and enjoyable for students.

KEY wORDs: Gymnastics; teaching method; game; high school; hygiene and assessment system. 
$\mathrm{F}$ ormado en la Escuela Central de profesores y profesoras de Gimnástica, a García Fraguas (1870-1909) no le terminaron de convencer los métodos de enseñanza empleados por muchos de sus maestros, razón que le llevó a buscar otras estrategias pedagógicas relacionadas con su materia, tanto en España como en el extranjero. Los pasos dados para configurar su propia acción docente le convirtieron en un distinguido e innovador profesor de Gimnástica.

La metodología que estableció en la segunda enseñanza se basó fundamentalmente en el juego como instrumento didáctico necesario en la escuela y en la instauración de ciertas rutinas de trabajo.

\section{Orden y disciplina}

García Fraguas estableció su propio sistema de ordenamiento en las clases de Gimnástica para favorecer la organización y rentabilizar el tiempo de aprendizaje con sus alumnos. En este sentido, el primer día de clase formaba grupos de cuarenta y cuatro alumnos y los agrupaba por estatura; primeramente seleccionaba a los cuatro mejores mozos y les asignaba una decena de alumnos para ser inscritos en una lista otorgada por él.

Durante la primera semana o algo más de tiempo, cuando era necesario, dependiendo de la capacidad y características de los estudiantes, dedicaba la segunda parte de la clase para dar la lección y realizar el registro higiénico pedagógico de cada alumno -empezaba por aquellos que presentaban algún achaque o defecto físico-, despidiendo a todos los demás que no necesitaba para el estudio.

Terminado el registro elegía, de entre los que realizaban los ejercicios con más belleza y resistencia, a otros dos encargados, designándolos como capitán y teniente de cada una de las secciones. Al cabo de un mes, después de haber finalizado las enseñanzas de marchas, carreras y saltos, escogía un gimnasta por cada cinco alumnos. Este cargo tenía una duración de un trimestre y al finalizar dicho periodo se hacía una selección entre alumnos nuevos y los que cesaban para otorgarles dos puestos de mayor categoría, Xistarca y Xofronista ${ }^{1}$, que se mantendrían hasta el final del curso; la posición de Xistarca se ganaba pasando unos ejercicios de agilidad y destreza, en este alumno delegaba el cometido de controlar la enseñanza y corrección de los ejercicios practicados por todos los demás; el Xofronista vigilaría el mantenimiento de la disciplina actuando en consecuencia. Para ocupar esta situación tendría que haber superado unas pruebas de fuerza y resistencia.

Los cargos se iban rotando a lo largo del curso, semanal o trimestralmente, dependiendo de cada uno de ellos. También podían perderse por faltas de asistencia.

En el supuesto de cometer una falta de disciplina, se trasmitía de cargo en cargo, por orden jerárquico hasta llegar al Xofronista si fuese necesario, quien se lo comunicaba al profesor en el hipotético caso de no haber sido corregida la conducta con anterioridad por ninguna otra figura de menor relevancia.

Empleando este tipo de organización, el profesor Fraguas fue capaz de gobernar clases de más de 200 alumnos «inculcándoles la emulación, la subordinación y la responsabilidad de sus actos» (García Fraguas, 1896: 391).

Las dos mayores dignidades y jefaturas del antiguo gimnasio griego. 


\section{Dinámica de la clase}

Preparaba sus clases teniendo en cuenta lo siguiente para la lección diaria: el sujeto de enseñanza -agrupados en secciones-, el lugar y el material a utilizar, además de atender a la normativa vigente en cada momento.

Sobre la lección pensaba que:

... su objeto es despertar, por medio del ejercicio, la actividad funcional del sistema circulatorio, respiratorio y excretorio, provocando la nutrición de todo el organismo y el desarrollo de las energías vitales, previo el equilibrio de las fuerzas nerviosas y el desarrollo de los músculos, de las cavidades torácica y abdominal, de la agudeza de los sentidos y de la conquista de una gradual fuerza, agilidad y destreza de todo el cuerpo humano (García Fraguas, 1896: 391).

La pedagógica forma de impartir sus lecciones le llevó a combinar ejercicios para que resultasen graduales, completas, útiles y amenas.

El profesor García Fraguas dividía la lección oficial en tres partes de cuarenta minutos cada una, y a continuación, después de formar y de pasar revista, encomendaba el trabajo a los encargados de las secciones, dedicándose también él a supervisar las tareas.

Los veinte minutos iniciales de la primera de las tres particiones los dedicaba a ejercitar movimientos espontáneos a pie firme; empezaba moviendo las piernas y acababa con ejercicios de cuello y cara para «los obesos y linfáticos» (García Fraguas, I896: 392). El resto del tiempo se dedicaba a practicar carreras con diferentes pasos y una carrera final.

Los segundos cuarenta minutos se destinaban al repaso de los ejercicios y conocimientos consumados en la lección anterior y a presentar algunos conceptos del tema siguiente.

De la tercera parte se consumían los primeros quince minutos para la explicación y el aprendizaje de un juego colectivo. El tiempo sobrante se empleaba para realizar uno o dos juegos conocidos, tomando parte en ellos también el profesor para estimularles y aplicar, en caso de necesidad, la oportuna acción correctora.

El final de la clase era anunciado por el bedel e inmediatamente el profesor daba la voz de: ¡Alto! para suspender las actividades, momento en el que los alumnos se colocaban por secciones con el encargado a la cabeza. El profesor se situaba enfrente, con el Xistarca a la derecha y el Xofronista a la izquierda, para inmediatamente desfilar los demás alumnos delante de ellos y saludar para despedirse. El Xistarca y el Xofronista se quedaban con el profesor para colocar el material y recibir las instrucciones correspondientes para la dirección de la siguiente clase.

Como síntesis, afirmó que «en toda lección debe tenerse presente la organización de la clase, la claridad y energía de las voces de mando, y atender especialmente los ejercicios de peligro y los de los débiles sin turbaciones ni brusquedades, pero con rapidez y abnegación» (García Fraguas, I896: 392).

Si analizamos su particular forma de organizar y dirigir las clases, en el modo de formar, de pasar revista, la voz de ¡alto! y la despedida, se observa un nítido tinte castrense. Es preciso recordar que García Fraguas, coincidiendo con el Dr. Lagrange, no era partidario de la gimnástica militar, así pues, entendemos que esta metodología fue utilizada únicamente para mantener en orden el elevado número 
de alumnos presentes en el aula y facilitar la observación de un comportamiento disciplinado en la misma.

\section{Reglas de higiene, indumentaria e instalaciones}

Según García Fraguas el profesor de Gimnástica estaba sujeto a unos preceptos higiénicos obligatorios, atinentes a mejorar el proceso de enseñanza-aprendizaje y evitar accidentes. Seguidamente exponemos de forma resumida las normas que habría de poner en práctica para ello:

- No practicar ejercicio físico inmediatamente antes o después de las dos principales comidas.

- Durante el invierno, los movimientos parciales debían sustituirse por otros más generales que implicasen desplazamientos.

- Iniciar la clase ejercitando los miembros inferiores para después practicar ejercicios de mayor intensidad. Al llegar a la mitad de la sesión la intensidad debía aminorar progresivamente hasta el término de la misma.

- La falta de aire para respirar y las palpitaciones del corazón advertían de la necesidad de guardar reposo.

- Los ejercicios de mucha intensidad serían interrumpidos con intervalos de descanso para favorecer la recuperación del cuerpo.

- La respiración de los alumnos tendría que ser profunda y extensa, alentándoles para realizar inspiraciones profundas y prolongadas, inspirando y espirando siempre por la nariz manteniendo la boca cerrada.

- Emplear con moderación todos aquellos ejercicios que requiriesen tener la posición de la cabeza hacia abajo.

- Durante la carrera se debía aconsejar llevar un ritmo de respiración adecuado.

- El canto, atendiendo a su ritmo e intensidad, era un magnífico ejercicio para trabajar la respiración.

- No ceñir la indumentaria al cuello ni al tórax durante la práctica deportiva.

- Ventilar los lugares donde se practicaba la Gimnástica, antes, durante y después de la práctica.

- Evitar los enfriamientos abrigándose inmediatamente después de la práctica corporal.

- Evitar las corrientes de aire.

- No beber agua fría cuando el cuerpo estuviese caldeado por la práctica de actividad física.

- Siempre que se pudiese sería recomendable el baño después del ejercicio. En su defecto sería adecuado lavarse con agua fría y secarse a continuación.

- Aumentar progresivamente la intensidad de las distintas tareas.

- No realizar ejercicios de fuerza ni ejercicios atléticos en la niñez, excepto para aquellos niños que tengan una estatura exagerada, ya que para estos adaptando las actividades les reportarían beneficios.

- Ejercitar los movimientos naturales aprovechando toda la amplitud de la articulación, pero sin llegar a forzar.

- Vincular el ritmo de los movimientos a la masa de los segmentos. 
- Realizar los ejercicios de flexión y extensión con suavidad, aguantando las posiciones durante unos segundos.

- Los ejercicios debían practicarse de forma simétrica, y trabajar igualmente los miembros inferiores y superiores.

- Practicar ejercicios de aproximación de los omoplatos y de resistencias horizontales para mejorar la fijación de la espalda.

- Realizar ejercicios que ejerciten los músculos del abdomen.

- Evolucionar desde ejercicios simples a otros más complejos, pasando por la descomposición de movimientos.

Muchas de las normas expuestas, con la excepción de algunas modificaciones y salvando las distancias entre las tendencias de la época y las actuales, son tenidas en cuenta por cada uno de los que hoy ejercemos como profesores de Educación Física.

Entendía Fraguas que la práctica deportiva debía realizarse de forma cómoda y segura. Para cumplir con este mandamiento había que utilizar la vestimenta adecuada y por ello recomendaba proveerse de: un traje interior de lana, una faja de Io $\mathrm{cm}$ de ancha y $3 \mathrm{~m}$ de larga y unos borceguíes ${ }^{2}$ de gamuza con suela de cuero o cáñamo y con poco tacón. Para las excursiones los caminantes debían ir provistos de un gorro y un bastón de cayada.

En verano orientaba al personal para utilizar ropa más ligera que en invierno y cambiarse de ropa después de realizar ejercicio físico.

Como amante de la educación física tampoco descuidaría el estado de los lugares en que se practicaba, exigiendo mantener las instalaciones en condiciones óptimas para que verdaderamente el ejercicio físico fuese saludable. Aconsejaba realizar las prácticas físicas en lugares abiertos, ventilados, confortables e iluminados, a ser posible por luz natural.

Idénticas características demandaba para los centros de enseñanza, además de reclamar un campo de juego cercano para promover el bienestar de los alumnos. Sostenía García Fraguas que tanto los patios como las salas de clase no eran los sitios idóneos para la práctica de la Gimnástica, y que cuando fuesen destinados para el uso de esta asignatura, el suelo no se debería cubrir con arena, serrín o cualquier otra materia que levantase polvo. Lo más acertado sería dejar el suelo desnudo y aplicar un riego antes de la práctica.

Teniendo conocimiento de que en los gimnasios privados se impartían clases a los alumnos de matrícula libre, nuestro interesado también determinó con detalle las condiciones de esos locales, por lo que nos parece interesante reflejarlo con sus propias palabras:

$\mathrm{I}^{\mathrm{a}}$. Situación: en las afueras de la ciudad, sobre un alto de roca caliza, accesible y con sótanos inhabitados que le aíslen de la humedad: mirando hacia el N. en los países fríos ${ }^{3}$, al NE. en los templados y al s. en los fríos, y procurando esté ventilado y bañado de luz. $2^{a}$. Forma y extensión: la más higiénica es un ángulo formado por dos pabellones con un solo piso y sótanos, entre cuyas paredes quede un patio espacioso para trabajar en días y horas apropiadas. La extensión corresponderá al número de alumnos, calculando las dimensiones y ventilación, de manera que todo ejercitante disponga de 20 metros cúbicos

2 Botas altas que se ajustan por medio de cordones o correas.

3 Consideramos que esto es un error, se referiría a países cálidos, no fríos. 
de aire por hora, cuando menos. Se recomienda la forma rectangular con los rincones cóncavos para el salón de ejercicios, provisto [sic] de buenos y suficientes ventiladores. $3^{\mathrm{a}}$. El material debe ser por su cantidad, calidad y aseo proporcionado á [sic] las más higiénicas y pedagógicas necesidades de la práctica (García Fraguas, I897: 4).

\section{Paseos y excursiones escolares}

Paralelamente al desarrollo de la Gimnástica sueca, considerada de carácter esencialmente pedagógico, a finales del xix surgió otro gran movimiento para transformar la educación física en todos los países, se trataba de la práctica de juegos corporales al aire libre y en pleno campo.

García Fraguas sería uno de los primeros en poner en marcha esta iniciativa, siendo valoradas positivamente y calificadas como ejemplares las excursiones y las prácticas de juegos escolares llevados a cabo en el Instituto de Salamanca -curso i893/94-. Mediante los paseos y excursiones buscaba el contacto de los alumnos con la naturaleza, disfrutando del aire puro y de todos sus beneficios y encantos.

El profesor abogó por la organización de excursiones desde todas las materias y enseñanzas, pues concebía que las específicas explicaciones -de Astronomía, Geología, Botánica y Agricultura, Mineralogía, Topografía y Agricultura, Mineralogía, Zoología, Matemáticas, etc.- resultarían más productivas sobre el terreno, a la vez que motivantes para los estudiantes. De la misma manera aprenderían a salvar los obstáculos encontrados durante la marcha y a apreciar las distancias.

Debido a la articulación del programa oficial de la asignatura de Gimnástica Fraguas quedaba obligado a realizar las excursiones establecidas por ley ${ }^{4}$. Siguiendo esta guisa, invitaba a empezar con paseos de cuatro kilómetros de ida y vuelta, para más adelante proceder con excursiones de un día completo, planificando los paraderos, las comidas, el tiempo de práctica y los descansos.

En concreto, seguía las siguientes normas para la organización de las salidas:

I. Anunciaban la excursión con una anticipación de veinticuatro horas, previa consulta del barómetro.

2. Exigía llevar ropas viejas y holgadas, cinturón elástico, zapatillas, sombrero ancho, un bastón de dos metros con contera, un cuaderno de apuntes 5 y una fiambrera con comida para los días de excursión completos. Quedaban prohibidos el agua ${ }^{6}$ y el vino.

3. La hora de salida quedaba estipulada en invierno a las ocho de la mañana y a las seis o las siete en otoño y primavera.

4. El lugar de salida sería el patio del instituto, desde donde ordenaba formar dos filas hasta salir de la ciudad.

5. Iniciada la excursión, fuera ya de la urbe, el primer kilómetro se recorría a paso ordinario, el segundo a paso largo, durante el tercero se comenzaba a correr, en el cuarto se elevaba un poco la intensidad de la carrera y en el último se retomaba el paso ordinario.

4 Dos al mes siempre que el tiempo lo permitiese. Ro de I de septiembre de I893.

s Los apuntes tomados por los alumnos eran de especial interés debido a que plasmaban sus propias impresiones. Se requería poner la fecha, el título de la excursión, escribir con claridad y «no decir inexactitudes ni exageraciones». García Fraguas (I896b).

6 Damos por hecho que durante el recorrido habría fuentes o riachuelos para poder beber. 
6. Finalizada la caminata se elegía un campo para jugar y entre los alumnos, por sufragio, seleccionaban los ejercicios o actividades a practicar por cada sección. A continuación el profesor se encargaba de vigilar y dirigir las prácticas además de jugar con ellos.

7. Después de jugar por secciones, solicitaba la reestructuración de grupos como los alumnos estimaran y continuaban jugando durante otros treinta minutos.

8. El juego se interrumpía para atender a otras clases, pues cuando la excursión duraba todo el día asistían más profesores, los cuales participaban de la naturaleza para impartir también sus materias.

9. El regreso se realizaba combinando dos kilómetros a paso ordinario y uno a la carrera, "permitiéndoles hablar ó [sic] cantar armonizadamente» (García Fraguas, 1896: 236).

\section{La utilización del juego como instrumento didáctico}

García Fraguas catalogó el juego como una herramienta fundamental para utilizar en los centros educativos. Su ideal se justificaba en interpretarlo como un medio capaz de despejar al individuo de su cansancio mental, reavivar el cuerpo y el alma y predisponer a la persona para la realización de otros trabajos. Teniendo presente este planteamiento, solicitó a las autoridades escolares la introducción de los juegos juveniles en los establecimientos de enseñanza -colegios, institutos y escuelas normales-y declaró que la escuela debía considerar el juego como: «una manifestación de la vida juvenil, igualmente saludable para el cuerpo, para el espíritu, para el corazón y para el sentimiento, capaz de producir un aumento de fuerza corporal y de destreza é [sic] influir favorablemente sobre la moral» (García Fraguas, I896: 297).

El juego entonces sería un bien preciado que se acoplaría perfectamente al carácter instintivo del niño, por esta razón García Fraguas lo consideró como el más natural e higiénico de los ejercicios. Definió el juego corporal como:

La rama ideal de la educación física encaminada al estudio de las aptitudes físicas, intelectuales y morales del discípulo, de su edad, sexo, país y época, con el objeto de despertar y perfeccionar las facultades ingénitas, provocándolas por el placer y la libertad, con el fin de mejorarlas ó consolidarlas por la práctica de diversiones y recreos regeneradores del cuerpo y del espíritu (García Fraguas, i897: 2).

El hecho de que fuese partidario del juego como instrumento didáctico para las clases de Educación Física no significa que rechazase otro tipo de trabajos. Es esencial

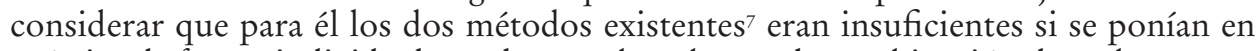
práctica de forma individual, por lo que abogaba por la combinación de ambos.

Luchó por la incorporación del juego pedagógico en la escuela, divulgando que:

... era la rama ideal de la educación física, hija del estudio de las aptitudes físicas, intelectuales y morales del alumno, de su país y de su época, con el objeto de despertar y perfeccionar las facultades humanas, provocando el impulso de la voluntad, animado

\footnotetext{
Se refería al método clásico centrado en la realización de movimientos regulados y prácticas con o en aparatos, y a otro más antiguo, casi en desuso, que recomendaba los juegos libres y ejercicios de fuerza y de destreza practicados al aire libre.
} 
por el placer, y educando su libertad, con el fin de perfeccionarle y mejorar la raza por medio del ejercicio corporal, administrado variada é [sic] inteligentemente en diversiones y recreos (García Fraguas, I896: 400).

Mediante este pensamiento trasladaba al maestro la responsabilidad de adecuarlo a las características de los alumnos en función de su edad, sexo, constitución, capacidad...

Atribuía al juego corporal un valor extraordinario, ya que suponía un recurso educativo muy eficaz para enseñar a los escolares nociones de deber, mando, subordinación, valor, heroísmo..., además de prepararles para afrontar victorias y derrotas.

Después de estudiar numerosas clasificaciones de diferentes autores, García Fraguas estableció la suya propia, dividiendo el juego corporal en: juegos físico-sociológicos, pedagógicos y aplicados. Los primeros comprenderían todos aquellos, tanto españoles como extrajeros, que se pudiesen practicar desde la niñez hasta la madurez; los pedagógicos albergarían los que se aplicasen al desarrollo de las facultades físicas, intelectuales y morales; y los aplicados harían referencia a los que se practicasen en las casas, patios, corrales, jardines, plazas, frontones y demás lugares de diversión y espectáculo.

El maestro de los juegos estaba obligado a ser un gran pedagogo para saber utilizarlos con maestría y dotarlos de un carácter educativo. Para conseguir esos fines Fraguas (1896: 403) recomendó seguir los consejos que el religioso P. Rosell había escrito en su obra en el s. XVIII:

- Sólo permitiréis á [sic] vuestros hijos y discípulos los juegos lícitos y honestos.

- Para la recreación se preferirán los entretenimientos y actos mediante los cuales se obtenga mayor utilidad.

- Se impedirá en los niños que las recreaciones degeneren una excesiva afición ó [sic] que se tomen con mucha seriedad.

- De los juegos y diversiones, eligiremos los más precisos y oportunos.

- Cuando la necesidad demande la elección de juegos, preferid los más sencillos.

- Acomodad las diversiones y los juegos á [sic] los tiempos y á [sic] la edad en que viven los niños.

- Apartad á [sic] los jugadores de ejercicios peligrosos y de futuras consecuencias.

\section{El sistema de evaluación}

Aceptando la evaluación como una actividad sistemática inherente en el proceso educativo, García Fraguas declaró literalmente en el tomo iII de su Tratado racional de Gimnástica que «el sujeto de la enseñanza [...], es un organismo cuya estructura debemos conocer antes de echarle á [sic] caminar» (I896: I40). Coherentemente, fiel a sus propias palabras, utilizaba la evaluación como punto de partida, además de emplearla para comprobar la progresión de sus estudiantes.

El marchamalero ${ }^{8}$, fruto de su celo profesional, creó un modelo de registro antropológico ${ }^{9}$ que propuso para evaluar a los alumnos, al que acompañó de unas reglas ${ }^{\mathrm{IO}}$ que servirían de guía para ser rellenado sin dificultad.

8 Natural de Marchamalo (Guadalajara).

9 Fue aceptado por el director general de Instrucción Pública -Sr. Vincenti-. García Fraguas, I896b: I43- I50).

1o Ibídem, pp. I5I-179. 
El arquetipo de registro ${ }^{\text {II }}$ planteado constaba de seis partes. En la primera se recogía la filiación del alumno; en la segunda los antecedentes morbosos donde se apuntaban las enfermedades familiares; en la tercera se reflejaba un examen anatómico, indicando la conformación general del cuerpo, el color de la piel, del pelo, de los ojos, todas las proporciones corporales -diámetros y circunferencias-, la talla y el peso; en la cuarta se anotaban los datos del examen fisiológico: apetito, régimen alimentario, digestiones, latidos cardíacos por minuto, pulsaciones de la arteria radial en un minuto, capacidad pulmonar, movimientos respiratorios por minuto y el estado de las excreciones sudoríficas, urinarias y fecales; en la quinta se registraban los datos del examen fisiológico-higiénico: la constitución, el temperamento, la idiosincrasia y las condiciones de la vivienda y aseo personal; por último, en la sexta habría que inscribir todo lo referente a las aptitudes físicas, el estado de la agilidad y de la destreza y las aptitudes intelectuales y morales.

Al término del registro metía un apéndice con espacios en blanco para escribir el estado de las inclinaciones artísticas, literarias, científicas, industriales y comerciales.

Por supuesto, en este documento debían aparecer los nombres de los profesores que lo hubiesen cumplimentado y con posterioridad enviarlo al Museo Antropológico Nacional.

No hace falta ser muy observador para darse cuenta de que era excelso el número de datos a considerar de cada uno de los alumnos. Según García Fraguas, la información recogida era suficiente para que el profesor conociera en profundidad a sus estudiantes y de esta manera poder comprobar sus progresos sin temor a equivocarse.

Afortunadamente los tiempos han cambiado y es evidente que en la actualidad este tipo de evaluación no tendría cabida en nuestro sistema educativo, pero, contextualizando esta labor estimadora de los conocimientos, actitudes y rendimiento de los alumnos (evaluar, según Diccionario de la RAE), juzgamos que para la época en que la asignatura de Gimnástica en los institutos daba sus primeros pasos en nuestro país, la presencia de este registro supuso una nueva asistencia para la pronta y eficaz estabilización de dicha materia, además de ser otra de las aportaciones personales de García Fraguas.

Recordamos que el método de evaluación establecido servía para informar al profesor y a los propios alumnos, en ningún momento para concretar una nota, pues la calificación era única, apto o no apto, y para ello se tenían en consideración también las asistencias.

Comprometido con el procedimiento de valoración de los escolares, debido a que había que medir la capacidad total de los pulmones para anotarlo en el registro antropométrico, ideó un espirómetro para facilitar el examen fisiológico. Lo costosos y frágiles que eran la mayoría de los modelos existentes fue lo que le condujo a investigar para confeccionar un espirómetro sencillo, económico y preciso.

El mecanismo se podía construir con una vejiga de cinco litros de cabida y una boquilla de caña o de metal. Funcionaba de la siguiente manera: después de una profunda inspiración se soplaba a través de la boquilla, que seguidamente era cerrada para evitar la salida de aire. Una vez hinchada se sumergía en una vasija llena de agua hasta el borde; la cantidad de agua vertida se recogía y se medía para apreciar la cantidad de líquido desalojado. Luego se vaciaba el aire de la vejiga y se llenaba de agua hasta que

" $\quad$ Fue modificado por la Dirección General de Instrucción Pública. Ibídem, pp. 333-336. 
hiciese rebosar de la vasija una cantidad de agua igual a la vertida cuando se sumergió hinchada de aire. Finalmente se vaciaba el líquido en un recipiente medidor y el total indicaba la cantidad de aire que salió de los pulmones.
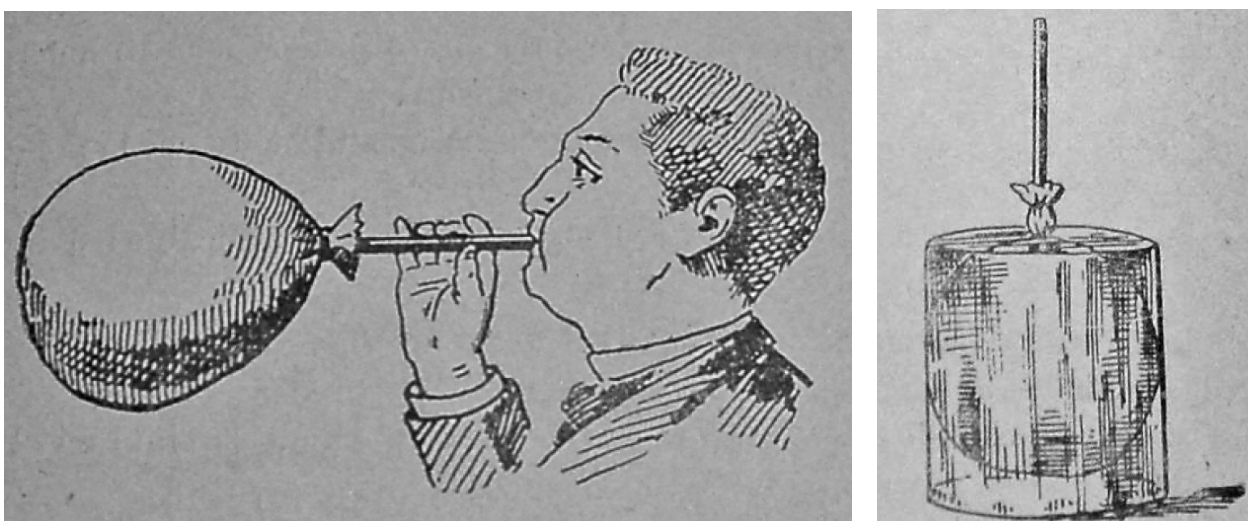

FIGs. I y 2. Primer y segundo tiempo del espirómetro de García Fraguas (1896: 160).

En el Programa ilustrado de advertencias y figuras de actitudes para las prácticas de la Gimnástica higiénica, sports, juegos y ejercicios medicinales García Fraguas (I897: 176-177) proponía también la prueba del sportsman y la carrera de obstáculos como lecciones para «examinar» a los alumnos de Gimnasia.

La prueba del sportsman requería una cierta habilidad en la carrera, el salto y la destreza por parte de los alumnos, puesto que englobaba un buen número de ejercicios. Era como una especie de circuito con diferentes postas o estaciones.

El desarrollo sería el siguiente:

I. ${ }^{\circ}$ Correr.

2. ${ }^{\circ}$ Franquear un obstáculo.

$3 .^{\circ}$ Lanzar con el pie un balón puesto en el suelo a un jugador situado a 20 metros.

$4 .^{\circ}$ Pasar de una estaca a otra sin ser alcanzado por objetos ${ }^{12}$ que arrojaban sobre él los jugadores colocados en hilera.

5. Recoger corriendo una bola y derribar una quilla.

6. Recoger corriendo una bola con la mano izquierda y lanzarla hasta el sitio en que se encuentre un jugador apostado.

7. Realizar el salto del carnero ${ }^{13}$ por encima de varios jugadores apostados.

8. ${ }^{\circ}$ Lanzar un balón, de modo que cayese al otro lado de una raya indicada.

9. ${ }^{\circ}$ Golpear una bola con un mallete ${ }^{\mathrm{t} 4} \mathrm{y}$ enviarla hasta una raya dibujada a cierta distancia.

Io. ${ }^{\circ}$ Tirar el arco y dar en el blanco.

II. ${ }^{\circ}$ Proseguir la carrera.

I2. ${ }^{\circ}$ Superar dos obstáculos.

12 Entendemos que se referiría a pelotas u otros materiales que no entrañasen peligro para los alumnos.

${ }_{13}$ Saltar por encima de un individuo que se encuentra agrupado apoyando los codos en la rodilla.

${ }_{14}$ Trozo de madera en forma de cuña. 
I3..$^{\circ}$ Ejecutar el pasa-ríos con cuerda ${ }^{15}$.

I4. $^{\circ} \quad$ Llegar hasta la meta.

La carrera de obstáculos fue considerada por García Fraguas como una lección más para poder valorar las capacidades de los escolares, aunque recomendaba que fuese puesta en práctica únicamente por jóvenes bien adiestrados. Se podía llevar a cabo durante varios días, a modo de competición, realizando clasificaciones según iban pasando de nivel al superar cada una de las pruebas (García Fraguas, I897: 177):

I. ${ }^{\circ}$ Pasar a través de una red al campo contrario.

2. ${ }^{\circ}$ Dar cinco pasos con zancos.

$3 .^{\circ}$ Atravesar en equilibrio las estacas.

$4 .^{\circ}$ Escalar por una plancha.

$5 .^{\circ}$ Descender por una percha.

6. ${ }^{\circ}$ Correr.

$7^{\circ}$ Cruzar nadando un río y llegar al final.

Sobre cualquiera de las dos pruebas, aunque con ejercicios diferentes, podemos decir que guardan parecido con ciertas actividades o ejercicios de algunas baterías utilizadas en la actualidad por profesores de Educación Física para la evaluación de sus alumnos.

\section{Conclusiones}

José Esteban García Fraguas completó su formación inicial como profesor en la Escuela Central de Gimnástica y paulatinamente fue construyendo su propio sistema de trabajo, perfilando, en base a sus posteriores estudios y a la experiencia, su metodología didáctica.

A pesar de no ser partidario de la Gimnástica Militar, podemos decir que el elevado número de alumnos existente le llevó a utilizar métodos disciplinarios relacionados con la milicia, con la mera intención de mejorar la organización de las mismas.

Llama la atención su forma de estructurar las clases de Gimnástica. Desde los inicios de la implantación de la asignatura en los planes de estudio de la segunda enseñanza combinó gran variedad de actividades: ejercicios individuales, colectivos, juegos, carreras, explicaciones teóricas..., incluyendo en todas sus clases un tiempo para repasar ejercicios aprendidos el día anterior.

Apoyándose en sus conocimientos como médico higienista, inculcó al alumnado la importancia de la higiene en general, así como la relevancia de la misma en las prácticas deportivas.

Conforme con el movimiento gimnástico surgido a finales del siglo decimonónico que incorporaba los juegos corporales al aire libre y las salidas al campo en la materia de Gimnástica, García Fraguas fue uno de los pioneros de su inclusión en la práctica docente, invitando a los alumnos a llevar una vida saludable disfrutando de la naturaleza.

Incansable defensor del juego como instrumento de aprendizaje, aprovechó el valor educativo del mismo, incorporándolo en sus sesiones como elemento motivador

is Implicaba colgarse de una cuerda para sobrepasar una superficie. 
y canalizador de la energía de los niños. Su convicción en los beneficios del juego le impulsó a solicitar a las autoridades escolares su integración en los planes de estudios.

Interesado en la forma de evaluar a sus discentes, llegó a idear un espirómetro casero de fácil manejo y bajo coste. Además propuso algunas pruebas para valorar la condición física de los escolares.

\section{Bibliografía}

AfUCm Libro de actas de la ECG. SG-I385. Archivo General de la Universidad Complutense de Madrid.

AGA Ejemplar del programa razonado de Gimnástica higiénica y juegos escolares para la Universidad, Instituto y Escuela Normal de Salamanca, I893. IDD (05) or6.000, caja 32/16308. Archivo General de la Administración.

AGA Expediente personal de la Facultad de Medicina de la Universidad Central. Legajo I39o, expediente 23, Sección de Universidades (Fondo de la Facultad de Medicina de la Universidad Central de Madrid). Archivo Histórico Nacional.

AGUCM Libro de registro de alumnos libres. AH-0384. Archivo General de la Universidad Complutense de Madrid.

AHPSA Libro de registro de matrícula de Gimnástica, legajo 15580. (Fondo Instituto Fray Luis de León). Archivo Histórico Provincial de Salamanca.

Aus Memorias de la Universidad de Salamanca (I894-1900). Salamanca: Establecimiento tipográfico de Francisco Núñez, I894. Rev. II78/9. Archivo de la Universidad de Salamanca.

Daniel Huerta, M. José (20II) El Profesor Fraguas y la Educación Física en Salamanca a finales del XIX. Trabajo de Grado no publicado. Universidad de Salamanca. Salamanca.

Daniel Huerta, M. José (2013) García Fraguas y la implantación de la Educación Física en España. Tesis doctoral no publicada. Universidad de Salamanca. Salamanca.

FERnández, S. (199I) La didáctica de la Gimnástica en el Siglo XIX. Revista BORDÓN, 43, 349-354.

GACETA DE MADRID (1894) La Enseñanza de la Gimnástica en los institutos, 78, 1065-1066.

García Fraguas, José Esteban (I89i) El juego de pelota como elemento gimnástico. El Heraldo de Madrid, 323, I.

García Fraguas, José Esteban (I892) Historia de la Gimnástica bigiénica y médica. Madrid: Establecimiento Tipográfico de Ricardo Fé.

García Fraguas, José Esteban (I893) Programa razonado de Gimnástica higiénica y juegos escolares para la Universidad, Instituto y Escuela Normal de Salamanca. Salamanca: Imprenta de Francisco Núñez Izquierdo.

García Fraguas, José Esteban (I894) Nuevo programa razonado de Gimnástica higiénica y juegos corporales para los trabajos de cuatro años de Educación Física. Madrid: Librería de la Viuda de Hernando y C.a.

García Fraguas, José Esteban (1895) El juego corporal i. La escuela moderna. Revista Pedagógica Hispano Americana, 50, 369-38I.

García Fraguas, José Esteban (I895) Memoria de higiene de la escuela y del régimen de la enseñanza. Valencia: Imprenta de Alpuente y Compañía.

García Fraguas, José Esteban (I896a) La higiene de la escuela y de la enseñanza. La escuela moderna. Revista Pedagógica Hispano Americana, 58, 37-54.

García Fraguas, José Esteban (I896b) Tratado Racional de Gimnástica y de los juegos corporales practicables sin aparatos y con ellos en las casas particulares, gimnasio, jardines $y$ en el campo y en las universidades, escuelas normales y municipales, institutos, academias, colegios, hospitales, sociedades de recreo, etc. Tomo III. Pedagogía General, Educación Física y Juegos corporales. Madrid: Imprenta Viuda de Hernando y C. . 
García Fraguas, José Esteban (1897) Programa ilustrado de advertencias y figuras de actitudes para las prácticas de Gimnasia higiénica, sports, juegos y ejercicios medicinales en los institutos, colegios, academias, escuelas, hospitales, habitaciones cerradas y abiertas, patios, corrales, jardines, plazas de recreo, etc. Madrid: Biblioteca de la Regeneración Física.

García Fraguas, José Esteban (1906) Higiene y educación. La Educación, I22, I.

López Gómez, S. (I88I) Breve reseña histórica de la Gimnástica en Europa. Sevilla: Juan Moyano.

Marín, E. (2009) D. Marcelo Santos Sanz Romo, iniciador y propagandista de la Educación Física en España: vida y obra. Tesis doctoral no publicada. Universidad de Alcalá. Alcalá de Henares.

Martínez Navarro, Anastasio (1983) Anotaciones a la historia de la Educación Física española en el siglo xix. Historia de la Educación. Revista Interuniversitaria, 2, I53-164.

Martínez Navarro, Anastasio (1995-96) Datos para la historia de una iniciativa fallida: La Escuela Central de Gimnasia. Historia de la Educación. Revista Interuniversitaria, I4-15, I25-I49.

Pastor Pradillo, José Luis (1995) La Educación Física en España: fuentes y bibliografía básicas. Alcalá de Henares: Servicio de Publicaciones de la Universidad de Alcalá.

Plana, C. (2002) La Educación Física en España durante las últimas décadas del siglo xIx: repercusiones de la circular de marzo de i894 fijando reglas sobre el planteamiento y desarrollo de la asignatura en los institutos. En Actas del Congreso Internacional de Historia de la Educación Física, 273-275.

Real Orden de i de septiembre de 1893, dictando reglas para el establecimiento de las cátedras de Gimnástica higiénica de los institutos provinciales. Gaceta de Madrid, núm. 253 de io de septiembre.

Sanz Romo, M. (1897) Manual de Gimnástica higiénica y juegos escolares. Madrid: Imprenta de los Sucesores de Cuesta.

SAnz Romo, M. (1898) Los ejercicios corporales o Gimnasia higiénica. Madrid: Imprenta de los Hijos de M. G. Hernández. 